\title{
Decentralized Group Sparse Beamforming for Multi-Cloud Radio Access Networks
}

\author{
Oussama Dhifallah $^{\dagger}$, Hayssam Dahrouj ${ }^{\ddagger}$, Tareq Y.Al-Naffouri ${ }^{\dagger}$ and Mohamed-Slim Alouini ${ }^{\dagger}$ \\ ${ }^{\dagger}$ Computer, Electrical and Mathematical Sciences and Engineering Division \\ King Abdullah University of Science and Technology (KAUST) \\ Email: \{oussama.dhifallah, tareq.alnaffouri, slim.alouini\}@kaust.edu.sa \\ $\ddagger$ Department of Electrical and Computer Engineering, Effat University \\ Email: hayssam.dahrouj@gmail.com
}

\begin{abstract}
Recent studies on cloud-radio access networks (CRANs) assume the availability of a single processor (cloud) capable of managing the entire network performance; inter-cloud interference is treated as background noise. This paper considers the more practical scenario of the downlink of a CRAN formed by multiple clouds, where each cloud is connected to a cluster of multiple-antenna base stations (BSs) via high-capacity wireline backhaul links. The network is composed of several disjoint BSs' clusters, each serving a pre-known set of single-antenna users. To account for both inter-cloud and intra-cloud interference, the paper considers the problem of minimizing the total network power consumption subject to quality of service constraints, by jointly determining the set of active BSs connected to each cloud and the beamforming vectors of every user across the network. The paper solves the problem using Lagrangian duality theory through a dual decomposition approach, which decouples the problem into multiple and independent subproblems, the solution of which depends on the dual optimization problem. The solution then proceeds in updating the dual variables and the active set of BSs at each cloud iteratively. The proposed approach leads to a distributed implementation across the multiple clouds through a reasonable exchange of information between adjacent clouds. The paper further proposes a centralized solution to the problem. Simulation results suggest that the proposed algorithms significantly outperform the conventional per-cloud update solution, especially at high signal-to-interference-plus-noise ratio (SINR) target.
\end{abstract}

\section{INTRODUCTION}

Cloud-radio access networks (CRANs) are considered a promising network architecture to meet the increasing demands in mobile data traffic and high energy efficiency for next generation wireless systems [1] [2]. In CRANs, joint signal processing is performed at multiple cloud computing centers (clouds) that are connected to several base stations (BSs) via high capacity backhaul links. As a result, large scale interference management and network power consumption reduction can be effectively achieved. Conventional strategies in CRANs assume the existence of a single cloud, and simply treat intercloud interference as part of the background noise. This paper considers the more realistic multi-cloud scenario. A major point in this paper is that a significant performance gain can be reached by accounting for both inter-cloud and intra-cloud interference, and jointly optimizing the beamforming vectors and the set of active BSs across the network.

\footnotetext{
Hayssam Dahrouj would like to thank Effat University in Jeddah, Saudi Arabia, for funding the research reported in this paper through the Research and Consultancy Institute.

The work of M.-S. Alouini was supported by the Qatar National Research Fund (a member of Qatar Foundation) under NPRP Grant NPRP 5-250-2-087. The statements made herein are solely the responsibility of the authors.

The authors would like to acknowledge the support provided by King Fahd University of Petroleum and Minerals (KFUPM) and King Abdullah University of Science and Technology (KAUST) for funding this work through the Research Institute project number EE002355.
}

This paper considers the downlink of a multi-CRAN scenario. The network is formed by a disjoint set of clusters of multi-antenna BSs, and each cloud is connected to one cluster via high capacity wireline backhaul links. Each cluster of BSs communicates with a pre-known set of single-antenna mobile users (MUs). Unlike the single-cloud model, the performance of the considered system becomes a function of both intercloud and intra-cloud interference, and depends on the set of active BSs within each cluster, as well as the beamforming vectors of the users across the network.

The problem considered in this paper is related in part to the recent literature on CRAN, especially to reference [2] which addresses the joint BS selection and beamforming design problem in the downlink of a single cloud scenario. The work in [2] considers the problem of minimizing the total network power consumption and solves the problem using techniques from compressive sensing literature. This paper is also related to reference [3] which addresses the user scheduling and beamforming vectors design problem by maximizing the weighted sum rate. Both references [2] and [3], however, assume a single-cloud scenario and do not account for inter-cloud interference. The problem in this paper is further related to the multicell beamforming problem studied in conventional cellular networks literature, e.g., [4] [5], which propose decentralized algorithms to solve the problem. The studies in conventional cellular networks [4] [5], however, assume that one user can be served by one BS only, unlike the CRAN architecture which is a user-centric architecture and allows each user to be served by one or more BSs at the same time.

The multi-CRAN setup considered in this paper is further related to the setup in [6] [7]. In [6], an iterative auction approach is used to solve the user-to-cloud association problem which maximizes the network-wide utility, under the general assumption of a pre-known set of active BSs and beamforming vectors. In [7], further, an iterative algorithm based on Majorization Minimization approach (MM) is proposed to jointly design the precoding matrix and the correlation matrix of the quantization noise to address the weighted sumrate maximization problem. The algorithm proposed in [7] is, however, centralized in nature, which is no longer feasible in practice, since otherwise, the clouds would require joint signal-processing and a high level of cloud-to-cloud backhaul communication.

Unlike all the aforementioned references, this paper considers a multi-CRAN and focuses on the problem of minimizing the total network power consumption subject to quality-ofservice requirements, so as to determine the sparse beamforming solution of each user across the network and the active set 
of BSs within each cluster of BSs. The main contribution of this paper is an algorithm that can be implemented in a decentralized fashion across the network, with a reasonable amount of information exchange between the clouds. The solution is based on Lagrangian duality theory using a dual decomposition approach, wherein the original optimization problem is decoupled into multiple and independent subproblems, each in charge of finding the local dual functions associated with each cloud. By allowing a limited information exchange between the coupled clouds, the proposed approach guarantees a feasible solution to the original problem at each iteration. The paper further proposes a centralized solution to the problem based on the group sparsity structure of the beamforming vectors. Simulation results show the performance improvement of the proposed solution as compared to the conventional per-cloud update solution.

\section{SyStem Model AND PROBLEM Formulation}

\section{A. System Model}

Consider the downlink of a multi-CRAN with $C$ clouds, where each cloud $c$ is connected to $B_{c}$ BSs via high-capacity wireline backhaul links. Each $\mathrm{BS} b$ is equipped with $N_{b}$ antennas. The network comprises $U$ single-antenna mobile users (MUs). Figure 1 illustrates an example of the considered network with 3 clouds, where each cloud is connected to 2 BSs. Let the set $\mathcal{C}$ with size $C=|\mathcal{C}|$ be the set of clouds, and

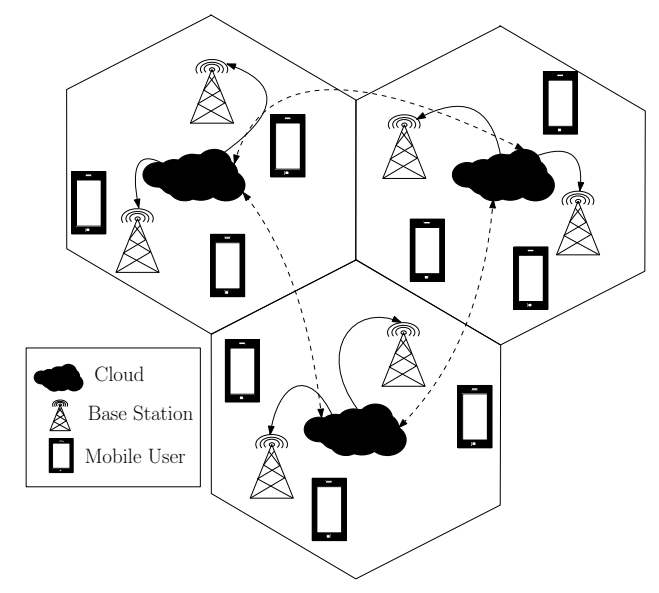

Figure 1. The architecture of multi-CRAN.

$\mathcal{U}_{c}$ be the set of users associated with one cloud $c \in \mathcal{C}$. Further, let $\mathcal{A}_{c}$ denote the set of active BSs connected to cloud $c$. Let $s_{u}$ be a complex scalar denoting the data symbol for user $u$, $\mathbf{w}_{c b u} \in \mathbb{C}^{N_{b}}$ be the beamforming vector at BS $b$ of cloud $c$ associated with user $u$ 's signal $s_{u}$, and $\mathbf{h}_{c b u} \in \mathbb{C}^{N_{b}}$ be the channel vector from BS $b$ of cloud $c$ to user $u$. The received signal $y_{u}^{c} \in \mathbb{C}$ of user $u$ in cloud $c$ can be written as:

$$
\begin{aligned}
y_{u}^{c} & =\sum_{b \in \mathcal{A}_{c}} \mathbf{h}_{c b u}^{H} \mathbf{w}_{c b u} s_{u}+\sum_{u^{\prime} \in \mathcal{U}_{c u}} \sum_{b \in \mathcal{A}_{c}} \mathbf{h}_{c b u}^{H} \mathbf{w}_{c b u^{\prime}} s_{u^{\prime}} \\
& +\sum_{c^{\prime} \neq c} \sum_{b^{\prime} \in \mathcal{A}_{c^{\prime}}} \sum_{u^{\prime} \in \mathcal{U}_{c^{\prime}}} \mathbf{h}_{c^{\prime} b^{\prime} u}^{H} \mathbf{w}_{c^{\prime} b^{\prime} u^{\prime}} s_{u^{\prime}}+n_{u},
\end{aligned}
$$

where $\mathcal{U}_{c u}=\mathcal{U}_{c} \backslash\{u\}$, and where $n_{u} \sim \mathcal{C N}\left(0, \sigma_{u}^{2}\right)$ represents the additive white Gaussian noise which is independent from the transmitted data symbols $s_{u}$.

Define the beamforming vector of user $u \in \mathcal{U}_{c}$ by $\mathbf{w}_{c u}=$ $\left[\mathbf{w}_{c b u}^{T}, \quad \forall b \in \mathcal{A}_{c}\right]^{T} \in \mathbb{C}^{N_{c}}$, which is the stacking of all beamforming vectors due to all active $\mathrm{BS} b \in \mathcal{A}_{c}$ associated with user $u$ 's signal $s_{u}$, where $N_{c}=\sum_{b \in \mathcal{A}_{c}} N_{b}$. Further, let the channel vector from the set of active BSs $b \in \mathcal{A}_{c}$ to user $u$ be $\mathbf{h}_{c u}=\left[\mathbf{h}_{c b u}^{T}, \quad \forall b \in \mathcal{A}_{c}\right]^{T} \in \mathbb{C}^{N_{c}}$. Therefore, the received signal at user $u$ in cloud $c$ can be rewritten as:

$$
\begin{aligned}
y_{u}^{c} & =\mathbf{h}_{c u}^{H} \mathbf{w}_{c u} s_{u}+\sum_{u^{\prime} \in \mathcal{U}_{c u}} \mathbf{h}_{c u}^{H} \mathbf{w}_{c u^{\prime}} s_{u^{\prime}} \\
& +\sum_{c^{\prime} \neq c} \sum_{u^{\prime} \in \mathcal{U}_{c^{\prime}}} \mathbf{h}_{c^{\prime} u}^{H} \mathbf{w}_{c^{\prime} u^{\prime}} s_{u^{\prime}}+n_{u} .
\end{aligned}
$$

\section{B. Problem Formulation}

Without loss of generality, assume that the average symbol power for user $u$ is unity, i.e. $E\left(\left|s_{u}\right|^{2}\right)=1$, and that the transmitted data symbols $s_{u}$ are independent from each other. Therefore, based on (2), the signal-to-interference-plus-noise ratio (SINR) of user $u$ served by cloud $c$ can be expressed as:

$$
\Gamma_{u}=\frac{\left|\mathbf{h}_{c u}^{H} \mathbf{w}_{c u}\right|^{2}}{\sum_{u^{\prime} \in \mathcal{U}_{c u}}\left|\mathbf{h}_{c u}^{H} \mathbf{w}_{c u^{\prime}}\right|^{2}+\sum_{c^{\prime} \neq c} \sum_{u^{\prime} \in \mathcal{U}_{c^{\prime}}}\left|\mathbf{h}_{c^{\prime} u}^{H} \mathbf{w}_{c^{\prime} u^{\prime}}\right|^{2}+\sigma_{u}^{2}} .
$$

One of the constraints of the optimization problem studied in this paper is the transmit power constraints of every BS $b$ connected to cloud $c$, given by:

$$
\sum_{u=1}^{U_{c}}\left\|\mathbf{w}_{c b u}\right\|_{\ell_{2}}^{2} \leq P_{c b}, \forall(b, c) \in\left(\mathcal{A}_{c} \times \mathcal{C}\right),
$$

where $P_{c b}$ is a given nominal maximum transmit power.

The objective function of the optimization problem studied in this paper is a generalization of the total network power consumption function studied in [2] in a single cloud setup. Using similar notations to the setup used in [2], this paper considers the total network power consumption across the multiple clouds, which can be written as follows:

$$
p(\mathcal{A}, \mathbf{w})=\sum_{c=1}^{C} \sum_{b \in \mathcal{A}_{c}} \sum_{u \in \mathcal{U}_{c}} \frac{1}{\nu_{b}}\left\|\mathbf{w}_{c b u}\right\|_{\ell_{2}}^{2}+\sum_{c=1}^{C} \sum_{b \in \mathcal{A}_{c}} P_{b}^{r},
$$

where $\mathcal{A}=\bigcup_{c=1}^{C} \mathcal{A}_{c}$ denotes the set of active BSs in the entire network, $\nu_{b}>0$ is the drain efficiency of the radio frequency (RF) power amplifier, and where $P_{b}^{r}>0$ denotes the relative wireline backhaul link power consumption which represent the difference between the power consumption of the active BSs and their corresponding transport link in active and sleep modes. The considered network power consumption model consists of the transmit power consumption of the active BSs and the relative wireline backhaul link power consumption.

The optimization problem studied in this paper considers the minimization of the multi-CRAN total network power consumption subject to SINR and transmit power constraints, which can be formulated as follows:

$$
\begin{array}{ll}
\min _{\mathbf{w}, \mathcal{A}} & \sum_{c=1}^{C} \sum_{b \in \mathcal{A}_{c}} \sum_{u \in \mathcal{U}_{c}} \frac{1}{\nu_{b}}\left\|\mathbf{w}_{c b u}\right\|_{\ell_{2}}^{2}+\sum_{c=1}^{C} \sum_{b \in \mathcal{A}_{c}} P_{b}^{r} \\
\text { s.t. } & \sum_{u \in \mathcal{U}_{c}}\left\|\mathbf{w}_{c b u}\right\|_{\ell_{2}}^{2} \leq P_{c b}, \forall(b, c) \in\left(\mathcal{A}_{c} \times \mathcal{C}\right) \\
& \Gamma_{u} \geq \delta_{u}, \forall(u, c) \in\left(\mathcal{U}_{c} \times \mathcal{C}\right),
\end{array}
$$

where the optimization is over the beamforming vectors $\mathbf{w}=\left[\mathbf{w}_{c b u}^{T}, \quad \forall(b, u, c) \in\left(\mathcal{A}_{c} \times \mathcal{U}_{c} \times \mathcal{C}\right)\right]^{T} \in \mathbb{C}^{N}$, where $N=\sum_{c=1}^{C} U_{c} N_{c}$, and over the set of active BSs $\mathcal{A}$, and where 
$\delta_{u}$ denotes the SINR target for user $u$. The above problem (4) is a mixed discrete and continuous optimization problem. Finding the global optimal solution for such a problem would likely require a combinatorial search, that is clearly infeasible for any sized network. Further, centralized solutions to the problem are no longer feasible, since otherwise, the clouds would require joint signal-processing and a high level of cloudto-cloud backhaul communication. Thus, instead of looking for global optimal solutions, the following section proposes a suboptimal decentralized algorithm to solve the problem. Simulation results suggest that the proposed decentralized algorithm converges to the centralized solution in a finite number of iterations. Furthermore, the proposed solution significantly outperforms the conventional per-cloud update solution.

\section{Decentralized Multi-Cloud Group Sparse BEAMFORMING ALGORITHM}

\section{A. Proposed Decentralized Solution}

This section proposes a decentralization solution to the total network power minimization problem (4), using a dual decomposition approach, first proposed in the context of multicell systems [5]. Specifically, we first start by introducing intercloud interference terms, and additional auxiliary variables so as to decouple the SINR constraints. The obtained optimization problem can then be solved locally at each cloud with the help of a dual decomposition approach [8] [9], thereby allowing the exchange of the local inter-cloud interference terms between the coupled clouds. Note that this approach decouples the original optimization problem into multiple and independent subproblems in charge of finding the local dual functions, controlled by a master problem responsible for updating the dual variables.

1) Problem Relaxation: First, define the inter-cloud interference terms $\xi_{c^{\prime} u}^{2}$ from an interfering cloud $c^{\prime}$ to user $u$ served by any cloud (different than $c^{\prime}$ ) as follows:

$$
\xi_{c^{\prime} u}^{2}=\sum_{u^{\prime} \in \mathcal{U}_{c^{\prime}}}\left|\mathbf{h}_{c^{\prime} u}^{H} \mathbf{w}_{c^{\prime} u^{\prime}}\right|^{2} .
$$

By relaxing the equality in the inter-cloud interference term $\xi_{c^{\prime} u}^{2}$ in (5) into an inequality, the total network power minimization problem (4) can be reformulated as:

$$
\begin{aligned}
& \min _{\mathbf{w}, \mathcal{A}, \boldsymbol{\xi}} \sum_{c=1}^{C} \sum_{b \in \mathcal{A}_{c}} \sum_{u \in \mathcal{U}_{c}} \frac{1}{\nu_{b}}\left\|\mathbf{w}_{c b u}\right\|_{\ell_{2}}^{2}+\sum_{c=1}^{C} \sum_{b \in \mathcal{A}_{c}} P_{b}^{r} \\
& \text { s.t. } \quad \sum_{u \in \mathcal{U}_{c}}\left\|\mathbf{w}_{c b u}\right\|_{\ell_{2}}^{2} \leq P_{c b}, \forall(b, c) \in\left(\mathcal{A}_{c} \times \mathcal{C}\right) \\
& \Gamma_{u} \geq \delta_{u}, \forall(u, c) \in\left(\mathcal{U}_{c} \times \mathcal{C}\right) \\
& \xi_{c^{\prime} u}^{2} \geq \sum_{u^{\prime} \in \mathcal{U}_{c^{\prime}}}\left|\sum_{b^{\prime} \in \mathcal{A}_{c^{\prime}}} \mathbf{h}_{c^{\prime} b^{\prime} u}^{H} \mathbf{w}_{c^{\prime} b^{\prime} u^{\prime}}\right|^{2}, \forall u \notin \mathcal{U}_{c^{\prime}}, \forall c^{\prime} \in \mathcal{C}, \\
& \Gamma_{u}=\frac{\left|\mathbf{h}_{c u}^{H} \mathbf{w}_{c u}\right|^{2}}{\sum_{u^{\prime} \in \mathcal{U}_{c u}}\left|\mathbf{h}_{c u}^{H} \mathbf{w}_{c u^{\prime}}\right|^{2}+\sum_{c^{\prime} \neq c} \xi_{c^{\prime} u}^{2}+\sigma_{u}^{2}}
\end{aligned}
$$

where the optimization is over the beamforming vectors, the set of active BSs in the entire network $\mathcal{A}$, and the inter-cloud interference vector $\boldsymbol{\xi}=\left[\xi_{c^{\prime} u}, \quad \forall u \notin \mathcal{U}_{c^{\prime}} \forall c^{\prime} \in \mathcal{C}\right]^{T} \in \mathbb{R}_{+}^{U^{\prime}}$, where $U^{\prime}=\sum_{c=1}^{C} \sum_{c^{\prime} \neq c} U_{c^{\prime}}$. Relaxing the inter-cloud interference constraints with inequality in the reformulated problem (6) is, in general, suboptimal as compared to (4) since the achieved
SINR is lower (i.e., more power is required to achieve the SINR target). However, the inequality turns out to be always tight.

Proposition 1. The original optimization problem (4) and the relaxed optimization problem (6) have the same optimal solution.

The proof of the above proposition follows by first showing by contradiction that the relaxed constraints are active at the optimal solution. Then, we can easily conclude the equivalence between the two optimization problems. The details are omitted due to space limitation. Note that the SINR constraints in problem (6) are still coupled across the different clouds, because of the inter-cloud interference terms, which still prevents the derivation of a decentralized strategy. However, using a similar approach to the decoupling strategy, first proposed in the multicell problem [5], (6) can be decoupled by introducing a set of auxiliary local variables at each cloud and a set of corresponding equality constraints. Problem (6) can then be reformulated as:

$$
\begin{array}{ll}
\min _{\mathbf{w}, \mathcal{A}, \tilde{\boldsymbol{\xi}}} & \sum_{c=1}^{C} \sum_{b \in \mathcal{A}_{c}} \sum_{u \in \mathcal{U}_{c}} \frac{1}{\nu_{b}}\left\|\mathbf{w}_{c b u}\right\|_{\ell_{2}}^{2}+\sum_{c=1}^{C} \sum_{b \in \mathcal{A}_{c}} P_{b}^{r} \\
\text { s.t. } & \sum_{u \in \mathcal{U}_{c}}\left\|\mathbf{w}_{c b u}\right\|_{\ell_{2}}^{2} \leq P_{c b}, \forall(b, c) \in\left(\mathcal{A}_{c} \times \mathcal{C}\right) \\
& \Gamma_{u}^{(c)} \geq \delta_{u}, \forall(u, c) \in\left(\mathcal{U}_{c} \times \mathcal{C}\right) \\
\xi_{c^{\prime} u}^{\left(c^{\prime}\right)^{2}} \geq \sum_{u^{\prime} \in \mathcal{U}_{c^{\prime}}}\left|\sum_{b^{\prime} \in \mathcal{A}_{c^{\prime}}} \mathbf{h}_{c^{\prime} b^{\prime} u}^{H} \mathbf{w}_{c^{\prime} b^{\prime} u^{\prime}}\right|^{2}, \forall u \notin \mathcal{U}_{c^{\prime}}, \forall c^{\prime} \in \mathcal{C} \\
\xi_{c^{\prime} u}^{\left(c^{\prime}\right)}=\xi_{c^{\prime} u}^{\left(c_{u}\right)}, \forall\left(u, c^{\prime}\right) \in\left(\mathcal{U} \times \overline{\mathcal{C}}_{u}\right), \\
\Gamma_{u}^{(c)}=\frac{\left|\mathbf{h}_{c u}^{H} \mathbf{w}_{c u}\right|^{2}}{\sum_{u^{\prime} \in \mathcal{U}_{c_{u}}}\left|\mathbf{h}_{c u}^{H} \mathbf{w}_{c u^{\prime}}\right|^{2}+\sum_{c^{\prime} \neq c} \xi_{c^{\prime} u}^{(c)}+\sigma_{u}^{2}}
\end{array}
$$

where $c_{u}$ denotes the cloud serving user $u, \overline{\mathcal{C}}_{u}=\left\{c \mid c \neq c_{u}\right\}$, and where the optimization is over the beamforming vectors, the set of active BSs $\mathcal{A}$, and the inter-cloud interference vector $\tilde{\boldsymbol{\xi}}=\left[\xi_{c^{\prime} u}^{(c)} \forall c \in\left\{c^{\prime}, c_{u}\right\} \forall u \notin \mathcal{U}_{c^{\prime}} \forall c^{\prime} \in \mathcal{C}\right]^{T} \in \mathbb{R}_{+}^{2 U^{\prime}}$. Note that by introducing the consistency constraints $\xi_{c^{\prime} u}^{\left(c^{\prime}\right)}=\xi_{c^{\prime} u}^{\left(c_{u}\right)}$ in (7), the SINR constraints become separable.

2) Subproblems Formulation: The distributed algorithm to solve problem (7) is obtained by adopting a dual decomposition approach. Specifically, we start by forming the partial Lagrangian over the consistency constraints as (i.e., by first accounting to the consistency constraints only):

$$
\begin{aligned}
\mathcal{L}(\mathbf{w}, \mathcal{A}, \boldsymbol{\xi}, \boldsymbol{\lambda}) & =\sum_{c=1}^{C} \sum_{b \in \mathcal{A}_{c}} \sum_{u \in \mathcal{U}_{c}} \frac{1}{\nu_{b}}\left\|\mathbf{w}_{c b u}\right\|_{\ell_{2}}^{2}+\sum_{c=1}^{C} \sum_{b \in \mathcal{A}_{c}} P_{b}^{r} \\
& +\sum_{u=1}^{U} \sum_{c^{\prime} \neq c_{u}} \lambda_{c^{\prime} u}\left(\xi_{c^{\prime} u}^{\left(c^{\prime}\right)}-\xi_{c^{\prime} u}^{\left(c_{u}\right)}\right),
\end{aligned}
$$

where $\lambda_{c^{\prime} u}$ denotes the Lagrange multiplier associated with the consistency constraint $\xi_{c^{\prime} u}^{\left(c^{\prime}\right)}=\xi_{c^{\prime} u}^{\left(c_{u}\right)}$, and $\lambda=$ $\left[\lambda_{c^{\prime} u}, \quad \forall u, \quad \forall c^{\prime} \in \overline{\mathcal{C}}_{u}\right]^{T} \in \mathbb{R}^{U^{\prime}}$.

Lemma 1. The right hand side term in the partial Lagrangian (8) can be rewritten as follows:

$$
J=\sum_{u=1}^{U} \sum_{c^{\prime} \neq c_{u}} \lambda_{c^{\prime} u}\left(\xi_{c^{\prime} u}^{\left(c^{\prime}\right)}-\xi_{c^{\prime} u}^{\left(c_{u}\right)}\right)=\sum_{c=1}^{C} \boldsymbol{\lambda}_{c}^{T} \boldsymbol{\xi}^{(c)},
$$

where the local dual variable $\boldsymbol{\lambda}_{c}=\left[\begin{array}{lll}\lambda_{c u^{\prime}} & \forall u^{\prime} & \notin\end{array}\right.$ 
$\left.\mathcal{U}_{c},-\lambda_{c^{\prime} u} \forall c^{\prime} \neq c \forall u \in \mathcal{U}_{c}\right]^{T}$ and the local inter-cloud interference vector $\boldsymbol{\xi}^{(c)}=\left[\xi_{c u^{\prime}}^{(c)} \forall u^{\prime} \notin \mathcal{U}_{c}, \xi_{c^{\prime} u}^{(c)} \forall c^{\prime} \neq c \forall u \in \mathcal{U}_{c}\right]^{T}$.

Proof: Define the set $S_{1}=\left\{\left(c, u^{\prime}\right) \mid c \in \mathcal{C}, u^{\prime} \notin \mathcal{U}_{c}\right\}$ ,and the set $S_{2}=\left\{(c, u) \mid c \in \mathcal{C}, u \in \mathcal{U}_{c}\right\}$. $S_{1}$ and $S_{2}$ are respectively equivalent to the following sets $S_{1}^{\prime}=\left\{(c, u) \mid u \in \mathcal{U}, c \neq c_{u}\right\}$ and $S_{2}^{\prime}=\left\{(c, u) \mid u \in \mathcal{U}, c=c_{u}\right\}$. By exploiting this equivalence, $J$ can be written as follows

$$
\begin{aligned}
J & =\sum_{u=1}^{U} \sum_{c^{\prime} \neq c_{u}} \lambda_{c^{\prime} u}\left(\xi_{c^{\prime} u}^{\left(c^{\prime}\right)}-\xi_{c^{\prime} u}^{\left(c_{u}\right)}\right) \\
& =\sum_{u=1}^{U} \sum_{c \neq c_{u}} \lambda_{c u} \xi_{c u}^{(c)}-\sum_{u=1}^{U} \sum_{c \neq c_{u}} \lambda_{c u} \xi_{c u}^{\left(c_{u}\right)} \\
& =\sum_{c=1}^{C}\left\{\sum_{u^{\prime} \notin \mathcal{U}_{c}} \lambda_{c u^{\prime}} \xi_{c u^{\prime}}^{(c)}-\sum_{u \in \mathcal{U}_{c}} \sum_{c^{\prime} \neq c} \lambda_{c^{\prime} u} \xi_{c^{\prime} u}^{(c)}\right\} \\
& =\sum_{c=1}^{C} \boldsymbol{\lambda}_{c}^{T} \boldsymbol{\xi}^{(c)} .
\end{aligned}
$$

Using the above Lemma, (7) can be transformed into a separable optimization problem, wherein the local dual functions, called $g_{c}\left(\boldsymbol{\lambda}_{c}\right)$, can be found separately at each cloud as follows:

$$
\begin{gathered}
g_{c}\left(\boldsymbol{\lambda}_{c}\right)=\min _{\mathbf{w}, \mathcal{A}, \boldsymbol{\xi}^{(c)}} \sum_{b \in \mathcal{A}_{c}} \sum_{u \in \mathcal{U}_{c}} \frac{1}{\nu_{b}}\left\|\mathbf{w}_{c b u}\right\|_{\ell_{2}}^{2}+\sum_{b \in \mathcal{A}_{c}} P_{b}^{r}+\boldsymbol{\lambda}_{c}^{T} \boldsymbol{\xi}^{(c)} \\
\text { s.t. } \sum_{u \in \mathcal{U}_{c}}\left\|\mathbf{w}_{c b u}\right\|_{\ell_{2}}^{2} \leq P_{c b}, \forall b \in \mathcal{A}_{c} \\
\Gamma_{u}^{(c)}=\frac{\left|\mathbf{h}_{c u}^{H} \mathbf{w}_{c u}\right|^{2}}{\sum_{u^{\prime} \in \mathcal{U}_{c u}}\left|\mathbf{h}_{c u}^{H} \mathbf{w}_{c u^{\prime}}\right|^{2}+\sum_{c^{\prime} \neq c} \xi_{c^{\prime} u}^{(c)^{2}}+\sigma_{u}^{2}} \geq \delta_{u} \\
\xi_{c u^{\prime}}^{(c)^{2}} \geq \sum_{u \in \mathcal{U}_{c}}\left|\sum_{b \in \mathcal{A}_{c}} \mathbf{h}_{c b u^{\prime}}^{H} \mathbf{w}_{c b u}\right|^{2}, \forall u^{\prime} \notin \mathcal{U}_{c} .
\end{gathered}
$$

For a fixed active set of BSs $\mathcal{A}$, the optimization problem (11) can be easily recast as a Second-Order Cone Programming (SOCP), which can then be solved using efficient numerical algorithms [10]. Therefore, the global optimal solution of the above optimization problem can be obtained by searching over all the possible active sets of BSs and selecting the set that provides the minimum cost value. Such approach to solve the optimization problem (11) grows exponentially with the number of BSs, which makes it unpractical. Since the minimization problem in formulation (11) is decoupled for every cloud, this paper rather proposes solving (11) using an iterative group sparse beamforming (GSBF) algorithm, first proposed in a single cloud setup [2]. Such approach provides a suboptimal solution to (11), but has a maximum number of iterations that is linear in the number of BSs. The GSBF approach relies on finding a tightest convex positively homogeneous lower bound [11] of the cost function, and then uses the MM algorithm [12] in order to induce group sparsity.

Proposition 2. The tightest convex positively homogeneous lower bound of the sparse representation of the cost function in (11) is given by

$$
\Omega\left(\mathbf{w}_{c}\right)=2 \sum_{b=1}^{B_{c}} \sqrt{\frac{P_{b}^{r}}{\nu_{b}}}\left\|\mathbf{w}_{c b}\right\|_{\ell_{2}}+\boldsymbol{\lambda}_{c}^{T} \boldsymbol{\xi}^{(c)}
$$

The proof of the above proposition uses steps similar to the ones used in [2], and hinges upon the fact that the second term in (12) is convex. Steps for determining the MM algorithm and the sparse representation of the cost function in (11) are also omitted in this paper, as they mirror the steps used in [2].

3) Master Problem: Solving (11) for every local dual function initially at every cloud $c$ requires the knowledge of the local dual variable $\boldsymbol{\lambda}_{c}$. Such variables are found by solving the master problem of the dual decomposition, which can be written as follows:

$$
\max _{\boldsymbol{\lambda}} g(\boldsymbol{\lambda})=\sum_{c=1}^{C} g_{c}\left(\boldsymbol{\lambda}_{c}\right)
$$

This dual problem can be solved iteratively and independently at each cloud using the subgradient method with the following update rule:

$$
\lambda_{c^{\prime} u}(i+1)=\lambda_{c^{\prime} u}(i)+\mu\left(\xi_{c^{\prime} u}^{\left(c^{\prime}\right)}-\xi_{c^{\prime} u}^{\left(c_{u}\right)}\right), \forall c^{\prime}, \forall u,
$$

where $\xi_{c^{\prime} u}^{\left(c^{\prime}\right)}-\xi_{c^{\prime} u}^{\left(c_{u}\right)}$ is a subgradient of the dual function $g$ at $\lambda_{\mathcal{C}^{\prime} u}$ [8], $i$ represents the iteration index and $\mu$ denotes the step size. As discussed earlier, for a fixed active set of BSs $\mathcal{A}$, the optimization problem (6) can be recast as a SOCP. Therefore, a diminishing step size, i.e. $\mu=\frac{K}{\sqrt{i}}$ where $K>0$, guarantees the convergence of the decentralized algorithm to the same solution of the centralized problem [13]. In this work, we only focus on a diminishing step size. As the simulations section suggest, the decentralized algorithm converges to the centralized solution in a finite number of iterations. Note that at each iteration of the subgradient algorithm, the coupled clouds need to exchange their current local inter-cloud interference terms, which is a reasonable level of information exchange.

4) Feasible Primal Solution: The dual decomposition approach consists of iterating between the optimization problem (11) and the update rule (14). At each iteration of this algorithm, the introduced auxiliary variables may not lead to a feasible solution for the original total network power minimization problem (4), i.e., $\xi_{c^{\prime} u}^{\left(c^{\prime}\right)} \neq \xi_{c^{\prime} u}^{\left(c_{u}\right)}$ during the intermediate iteration. To reach a set of feasible solution at each iteration, we propose using fixed average inter-cloud interference terms defined as follows:

$$
\xi_{c^{\prime} u}=\frac{1}{2}\left(\xi_{c^{\prime} u}^{\left(c^{\prime}\right)}+\xi_{c^{\prime} u}^{\left(c_{u}\right)}\right), \forall u \notin \mathcal{U}_{c^{\prime}}, \forall c^{\prime} \in \mathcal{C} .
$$

Then, resolve the optimization problem (11) for fixed average inter-cloud interference terms. Note that the obtained solution remains suboptimal, yet a feasible, solution to the original problem (4).

5) Iterative Decentralized Algorithm: The proposed decentralized solution requires to iterate between two levels. At the first level, multiple and independent subproblems are solved locally. At the second level, there is a master problem responsible for updating the dual variables. At each iteration, a solution of feasible beamforming vectors and active set of BSs is estimated locally. The proposed iterative Decentralized MultiCloud Group Sparse Beamforming (I-DMCGSB) algorithm is summarized in Table (1).

\section{B. Centralized Solution}

In this section, we derive a centralized solution for the optimization problem (4). To this end, we assume that all the processing is performed in a shared processor connecting all the clouds. The total power minimization problem (4) can be 
$\overline{\text { Algorithm } 1 \text { The iterative Decentralized Multi-Cloud Group }}$ Sparse Beamforming (I-DMCGSB)

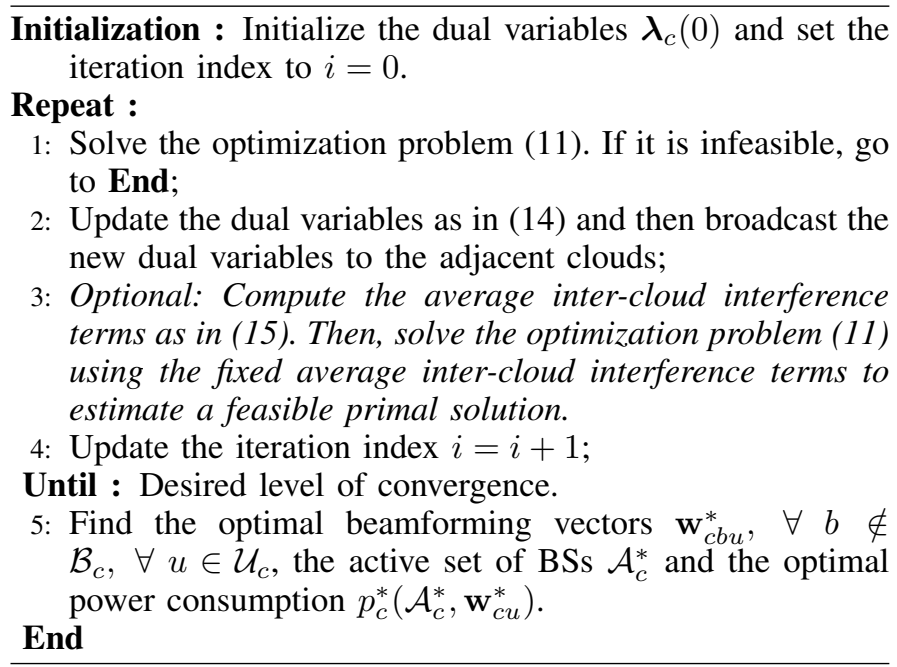

reformulated as

$$
\begin{aligned}
& \min _{\mathbf{w}^{\prime}, \mathcal{A}} \sum_{b \in \mathcal{A}} \sum_{u \in \mathcal{U}} \frac{1}{\nu_{b}}\left\|\mathbf{w}_{b u}\right\|_{\ell_{2}}^{2}+\sum_{b \in \mathcal{A}} P_{b}^{r} \\
& \text { s.t. } \quad \sum_{u \in \mathcal{U}}\left\|\mathbf{w}_{b u}\right\|_{\ell_{2}}^{2} \leq P_{b}, \forall b \in \mathcal{A} \\
& \Gamma_{u}^{\prime}=\frac{\left|\mathbf{h}_{u}^{\prime H} \mathbf{w}_{u}^{\prime}\right|^{2}}{\sum_{u^{\prime} \neq u}\left|\mathbf{h}_{u}^{\prime H} \mathbf{w}_{u^{\prime}}^{\prime}\right|^{2}+\sigma_{u}^{2}} \geq \delta_{u}, \forall u \in \mathcal{U} \\
& \quad\left\|\mathbf{w}_{b u}\right\|_{\ell_{2}} \leq 0, \forall(u, b) \in\left(\mathcal{U} \times \overline{\mathcal{A}}_{u}\right),
\end{aligned}
$$

where $\mathbf{w}_{u}^{\prime}$ denotes the beamforming vector of user $u$ which is defined as the stacking of all beamforming vectors used by the set of active BSs $b \in \mathcal{A}$ to serve user $u$, and $\mathbf{h}_{u}^{\prime}$ represents the channel vector from the entire set of active BSs $b \in \mathcal{A}$ to user $u, \mathbf{w}_{b u}$ denotes the beamforming vector at BS $b$ for user $u, N^{\prime}=U \sum_{b \in \mathcal{A}} N_{b}, \overline{\mathcal{A}}_{u}$ denotes the active set of BSs that can not serve user $u$ and $P_{b}$ denotes the maximum transmit power of BS $b$. In order to guarantee the equivalence between the centralized formulation and the original total network power minimization problem (4), we introduce additional inequality constraints $\left\|\mathbf{w}_{b u}\right\|_{\ell_{2}} \leq 0, \forall(u, b) \in\left(\mathcal{U} \times \overline{\mathcal{A}}_{u}\right)$, so as to impose that the beamforming vectors form BS $b$ to user $u$ connected to different cloud is equal to zero. Thus, solving the optimization problem (16) is equivalent to solving the original problem (4).

The introduced constraints in (16) are Second-Order Cone (SOC) constraints. The above optimization problem can, therefore, be recast as a SOCP for a fixed active set of BSs $\mathcal{A}$, which can then be solved efficiently [10]. In order to reduce the complexity, iterative GSBF algorithm can be used which provides a suboptimal solution to (16) with low computational complexity.

\section{Per-Cloud Update Solution}

Conventional CRANs operate on a per-cloud basis; intercloud interference is treated as background noise. The baseline approach to solve (4) then consists of iteratively solving the total network power consumption minimization problem on a per-cloud basis, where in each iteration, the cloud under consideration solves the problem locally while treating inter- cloud interference terms as part of the background noise. Specifically, in iteration $i$, cloud $c$ determines its set of beamformers and active set of BSs for fixed inter-cloud interference. The resulting solution then affects the solution at cloud $c^{\prime}$ in the following iteration, as the inter-cloud interference terms need to be updated. Such solution, called per-cloud update solution, iteratively proceeds in updating the solution of each cloud, in view of the other clouds' solutions.

\section{Simulation RESUlts}

This section investigates the performance of the proposed algorithms. Consider a two-cloud CRAN, where each cloud is connected to two BSs, i.e., $B_{1}=B_{2}=2$. Each cluster of BSs serves two single-antenna MUs. Each BS is equipped with five antennas. We assume that the BSs and MUs in the first cloud are uniformily and independently distributed in the square region $[02000] \times[02000]$ meters. In the second cloud, the BSs and MUs are uniformily and independently distributed in the square region [2000 4000] $\times[02000]$ meters. The channel vectors from the BSs to the MUs are generated assuming a distance-dependent path loss $L\left(d_{b u}\right)=128.1+37.6 \log _{10}\left(d_{b u}\right)$, and Rayleigh fading component, where $d_{b u}$ denotes the distance between BS $b$ and user $u$ in kilometers. The noise power spectral density is $\sigma_{u}^{2}=-96 \mathrm{dBm} \forall u$. The transmit antenna power gain is set to $9 \mathrm{dBi}$. We set the initial dual variable to $\lambda_{c u}(0)=0.01, \forall u \in \mathcal{U}, \forall c \in \overline{\mathcal{C}}_{u}$. The drain efficiency of the radio frequency power amplifier is set to $\nu_{b}=0.25, \forall b$. The relative wireline backhaul link power consumption is set to $P_{b}^{r}=2+l_{b}, \forall b$, where $l_{b}$ denotes the number of $\mathrm{BS} b$ in cloud $c$. We set the maximum transmit power of BS $b$ in cloud $c$ to $P_{c b}=1 W$.

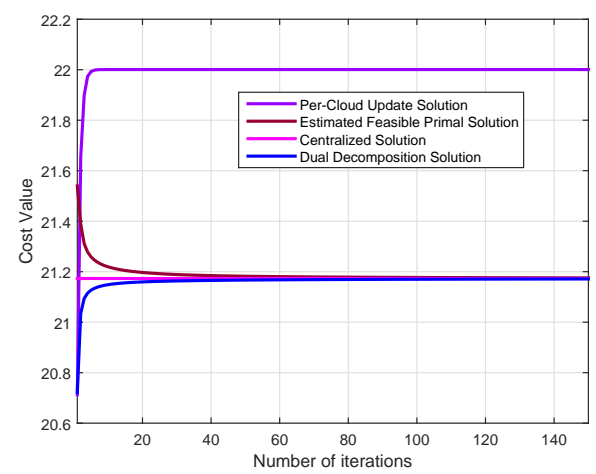

Figure 2. Convergence behavior of the I-DMCGSB and the conventional per-cloud update algorithms with SINR target $=5 \mathrm{~dB}$ and $K=2$.

First, we set the SINR target to $5 \mathrm{~dB}$ and the step size $\mu=\frac{2}{\sqrt{i}}$. Figure 2 plots the network power consumption (in Watts) versus the number of iterations so as to illustrate the convergence behavior of the proposed I-DMCGSB algorithm and the conventional per-cloud update algorithm. The figure shows that the estimated feasible primal solution is a tight upper bound on the solution of the original optimization problem. It can be noticed that our proposed decentralized solution I-DMCGSB algorithm converges to the centralized solution in less than 100 iterations. Figure 2 particularly shows that the proposed joint optimization solution (centralized and decentralized) significantly outperforms the conventional percloud update solution.

Then, we compare the energy efficiency of the proposed and the conventional per-cloud update decentralized algo- 


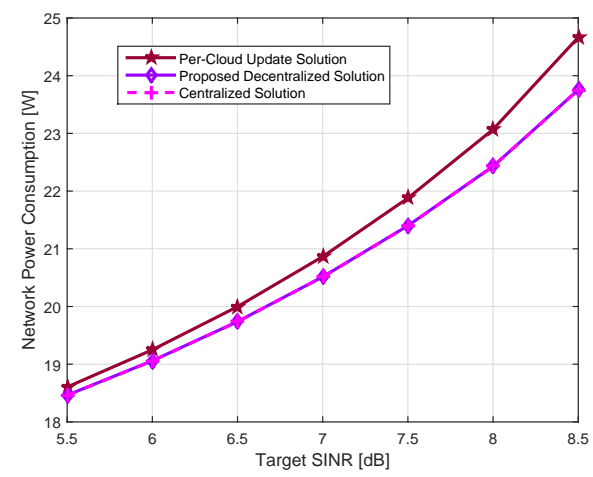

Figure 3. Total network power consumption versus SINR target with $K=2$.

rithms. Figure 3 plots the total network power consumption as a function of SINR target. The figure shows that the proposed decentralized solution has the same performance as the centralized solution for all values of SINR target. Furthermore, the proposed solution significantly outperforms the per-cloud update solution, especially at high SINR target.

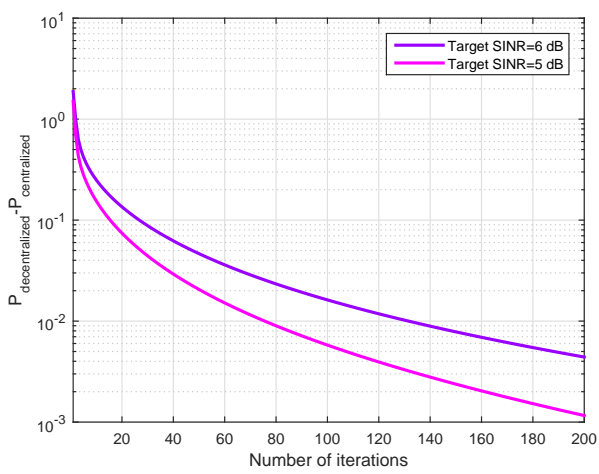

Figure 4. Suboptimality versus iteration number for dual decomposition with different SINR targets and $K=2$.

Figure 4 plots the difference between the estimated feasible primal solution, denoted by $P_{\text {decentralized, obtained using the }}$ average inter-cloud interference terms and the solution of the centralized algorithm, denoted by $P_{\text {centralized, as a function of }}$ the iteration index for two values of SINR targets. It can be noticed that the convergence of the I-DMCGSB algorithm is faster for low SINR target. This result can be explained by the fact that increasing the SINR target leads to higher network power consumption, and so more iterations are needed to achieve the desirable convergence.

Finally, to investigate the impact of changing the step size on the convergence speed of the algorithm, Figure 5 shows $P_{\text {decentralized }}-P_{\text {centralized }}$ as a function of the iteration index for different step sizes under a SINR target of $4 \mathrm{~dB}$. The figure shows that the convergence speed of the proposed decentralized I-DMCGSB algorithm is sensitive to the used step size. Figure 5 especially shows how a well chosen step size may lead to a significant improvement in the convergence speed of the algorithm.

\section{CONCLUSION}

In this paper, a downlink multi-cloud radio access network is considered, where each cloud is connected to several multiple-antenna BSs via high capacity backhaul links. The

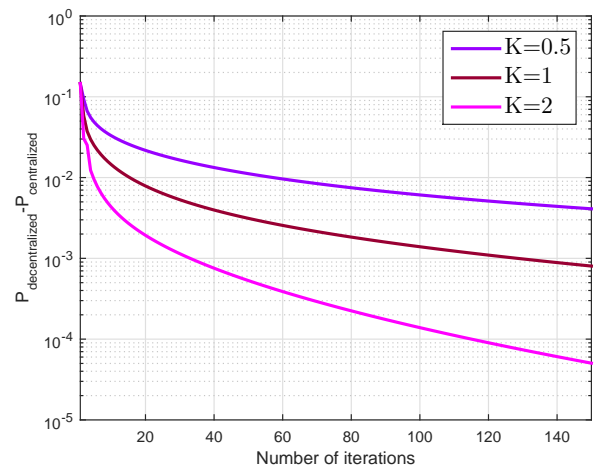

Figure 5. Suboptimality versus iteration number for dual decomposition with different step-sizes and fixed SINR target $=4 \mathrm{~dB}$.

paper proposes a decentralized algorithm to solve the total network power minimization problem using a dual decomposition approach. A feasible active set of BSs and beamforming vectors can be obtained locally at each iteration by allowing a limited information exchange between the adjacent clouds. Simulation results show that the proposed decentralized algorithm converges to the centralized solution in a finite number of iterations, and the proposed algorithms significantly outperform the conventional per-cloud update solution, especially at high SINR target.

\section{REFERENCES}

[1] J. Andrews, S. Buzzi, W. Choi, S. Hanly, A. Lozano, A. Soong, and J. Zhang, "What will $5 \mathrm{~g}$ be?" Selected Areas in Communications, IEEE Journal on, vol. 32, no. 6, pp. 1065-1082, June 2014.

[2] Y. Shi, J. Zhang, and K. Letaief, "Group sparse beamforming for green cloud-ran," Wireless Communications, IEEE Transactions on, vol. 13, no. 5, pp. 2809-2823, May 2014.

[3] B. Dai and W. Yu, "Sparse beamforming and user-centric clustering for downlink cloud radio access network," CoRR, vol. abs/1410.5020, 2014. [Online]. Available: http://arxiv.org/abs/1410.5020

[4] H. Dahrouj and W. Yu, "Coordinated beamforming for the multicell multi-antenna wireless system," Wireless Communications, IEEE Transactions on, vol. 9, no. 5, pp. 1748-1759, May 2010.

[5] A. Tolli, H. Pennanen, and P. Komulainen, "Decentralized minimum power multi-cell beamforming with limited backhaul signaling," Wireless Communications, IEEE Transactions on, vol. 10, no. 2, pp. 570580, February 2011.

[6] H. Dahrouj, T. Y. Al-Naffouri, and M.-S. Alouini, "Distributed cloud association in downlink multicloud radio access networks," CoRR, vol. abs/1503.00267, 2015. [Online]. Available: http://arxiv.org/abs/1503.00267

[7] S.-H. Park, O. Simeone, O. Sahin, and S. Shamai, "Inter-cluster design of precoding and fronthaul compression for cloud radio access networks," Wireless Communications Letters, IEEE, vol. 3, no. 4, pp. 369-372, Aug 2014.

[8] S. Boyd, L. Xiao, A. Mutapcic, and J. Mattingley, "Notes on decomposition methods: course reader for convex optimization II, Stanford," 2007, available online: http://www.stanford.edu/class/ee364b/.

[9] D. Palomar and M. Chiang, "A tutorial on decomposition methods for network utility maximization," Selected Areas in Communications, IEEE Journal on, vol. 24, no. 8, pp. 1439-1451, Aug 2006.

[10] S. P. Boyd and L. Vandenberghe, Convex Optimization. Cambridge University Press, 2004.

[11] G. Obozinski and F. Bach, "Convex Relaxation for Combinatorial Penalties," ArXiv e-prints, May 2012.

[12] D. R. Hunter and K. Lange, "A tutorial on MM algorithms," Amer.Statistician, pp. 30-37, 2004.

[13] S. Boyd and A. Mutapcic, "Subgradient methods: course reader for convex optimization II, Stanford," 2007, available online: http://www.stanford.edu/class/ee364b/. 